Presented at $22^{\text {st }}$ Digital Avionics Systems Conference, October 14-16, 2003, Indianapolis, IN

\title{
THE APPLICATION OF LIDAR TO SYNTHETIC VISION SYSTEM INTEGRITY
}

\author{
Jacob L. Campbell, Maarten Uijt de Haag, Ananth Vadlamani, Ohio University, Athens, Ohio \\ Steve Young, NASA Langley Research Center, Hampton, Virginia
}

\begin{abstract}
One goal in the development of a Synthetic Vision System (SVS) is to create a system that can be certified by the Federal Aviation Administration (FAA) for use at various flight criticality levels. As part of NASA's Aviation Safety Program, Ohio University and NASA Langley have been involved in the research and development of real-time terrain database integrity monitors for SVS. Integrity monitors based on a consistency check with onboard sensors may be required if the inherent terrain database integrity is not sufficient for a particular operation. Sensors such as the radar altimeter and weather radar, which are available on most commercial aircraft, are currently being investigated for use in a real-time terrain database integrity monitor. This paper introduces the concept of using a Light Detection And Ranging (LiDAR) sensor as part of a real-time terrain database integrity monitor. A LiDAR system consists of a scanning laser ranger, an inertial measurement unit (IMU), and a Global Positioning System (GPS) receiver. Information from these three sensors can be combined to generate synthesized terrain models (profiles), which can then be compared to the stored SVS terrain model. This paper discusses an initial performance evaluation of the LiDAR-based terrain database integrity monitor using LiDAR data collected over Reno, Nevada. The paper will address the consistency checking mechanism and test statistic, sensitivity to position errors, and a comparison of the LiDAR-based integrity monitor to a radar altimeter-based integrity monitor.
\end{abstract}

\section{Introduction}

SVS can be defined as a perspective pilot display that depicts the current pilot view of the world given a database of the area and knowledge of the current aircraft state. The use of SVS to improve a pilot's Situational Awareness (SA) is one of the main goals outlined by NASA for commercial and business aircraft SVS use [1]. As with any aircraft system, the SVS must be designed to meet levels of accuracy, integrity, availability, and continuity dictated by the criticality of the system with respect to the safety of flight. Ohio University's Avionics Engineering Center and NASA Langley Research Center are developing methods which will improve the integrity of Digital Elevation Models (DEMs) used by SVS. This is being done by the inclusion of real-time integrity monitors for the SVS DEMs.

A Downward Looking Integrity Monitor (DLIM) was first proposed in [2]. The DLIM increased the integrity of a terrain database using sensors that are found on most commercial transport aircraft. A second method, which involves the use of forward looking weather radar in terrain mapping mode, is also being considered [3].

It has been shown in [4] that with the relatively large error distributions assigned to currently available DEMs along with limitations to the radar altimeter performance provides integrity in the vertical direction; however the horizontal integrity of the DEMs is not improved significantly. As new remote sensing technologies are invented and refined, it is interesting to apply these new technologies to the DLIM to further improve the DEM integrity monitor. This paper presents the use of airborne LiDAR technology to generate DEMs and as part of a DLIM.

\section{Downward Looking Integrity Monitor}

As has been discussed in previous papers [2] [3] [4] [5], the integrity of the terrain database in a SVS can be monitored given a method to compare the agreement between the database and the actual terrain. As proposed in [2], a synthesized terrain profile can be generated using Differential GPS (DGPS) and radar altimeter data then compared to the profile traced in the terrain database. To be more specific, the synthesized terrain profile, 
$h_{S Y N T}\left(t_{i}\right)$, is generated by subtracting the aircraft height Above Ground Level (AGL) from the aircraft height above Mean Sea Level (MSL). Figure 1 illustrates how the synthesized terrain profile is generated using the height AGL, $h_{R A D}$, provided by the Radar Altimeter, and the height MSL, $h_{D G P S}$, provided by the DGPS.

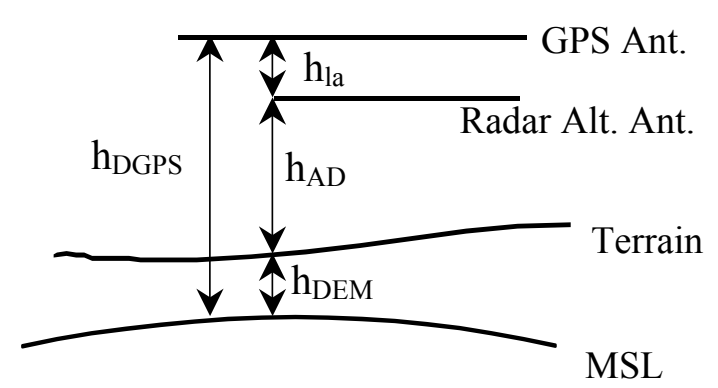

Figure 1. Synthesized Terrain Profile

The disparity is the metric used in the integrity monitor. It is generated by subtracting the terrain database height, $h_{D E M}\left(\operatorname{lat}\left(t_{i}\right), \operatorname{lon}\left(t_{i}\right)\right)$, from the synthesized height, $h_{S Y N T}\left(t_{i}\right)$, to form the disparity $p\left(t_{i}\right)$ as seen in equation (1)

$$
p\left(t_{i}\right)=h_{S Y N T}\left(t_{i}\right)-h_{D T E D}\left(\operatorname{lat}\left(t_{i}\right), \operatorname{lon}\left(t_{i}\right)\right)
$$

The agreement in a sequence of disparities is then quantified by the Mean Squared Error (MSE).

$$
M S E=\frac{1}{N} \sum_{i=1}^{N} p^{2}\left(t_{i}\right)
$$

The MSE is scaled by the standard deviation of the normal operation errors, $\sigma_{T}$, to form the following test statistic $T$ [2].

$$
T=\frac{N}{\sigma_{T}^{2}} M S E=\frac{1}{\sigma_{T}^{2}} \sum_{i=1}^{N} p^{2}\left(t_{i}\right)
$$

It was shown in [2] that using $\mathrm{N}=50$ independent disparities, a test statistic threshold, $T_{d}$, of 96 would lead to a probability of false alarm $\left(\mathrm{P}_{\mathrm{FFD}}\right)$ of $10^{-4}$. The minimum detectable bias is dependent on the standard deviation of the disparities, and the time-to-alert is defined as the time it takes to make $N$ independent disparity measurements.

In this paper the LiDAR is used to generate the synthesized profile, $h_{S Y N T}$.

\section{Light Detection and Ranging (LiDAR) Sensor}

A LiDAR system consists of a laser scanner, Inertial Measurement Unit (IMU) and a Global Positioning System (GPS) receiver [6]. Combining the data from these sensors allows for the precise earth-referenced (10-20cm accuracy [7]) measurement of the point illuminated by the laser scanner. Most of today's LiDAR systems are designed for terrain mapping and operate in the near infra-red spectrum and their eye-safety classification ranges from Class 1 to Class $4 \mathrm{~b}$ [8]. This section is designed to provide an overview of the characteristics of an Airborne LiDAR sensor. For more detailed information see [6] [7] [9] [10] [11] [12].

\section{LiDAR Error Budget (Post-processed Data)}

There are three major sensor components in a LiDAR system- the laser scanner, the IMU, and the GPS receiver. The laser scanner sweeps side to side, making hundreds of angle encoded range measurements per sweep. The GPS and IMU provide position and orientation information respectively. Typically, the aircraft GPS data is combined with data from a GPS reference station to achieve centimeter-level Kinematic GPS position solutions. IMU data is often processed with the Kinematic GPS solution to provide a higher update rate position solution, and to solve for unknown biases and drifts in the IMU. The errors from each of these sensors contribute to the laser point measurement error. This relationship will be explored to produce a new vertical error distribution for the DLIM.

\section{Laser Scanner Sensor Errors}

Most topographic laser scanners in use today modulate the laser using a pulsing scheme to measure the range and an encoder or galvanometer to measure the laser scan angle. Thus, the laser scanner sensor errors can be broken into two categories- ranging errors and scan angle errors. 
Ranging error is defined by (4) to be a function of the pulse rise time, $t_{\text {rise }}$, the signal-to-noise ratio (SNR), and the speed of light, c [7].

$$
\sigma_{R}=\frac{\mathrm{c}}{2} t_{\text {rise }} \frac{1}{\sqrt{\mathrm{SNR}}}
$$

A typical pulse rise time is $1 \mathrm{~ns}$, and the SNR can vary greatly depending on the target reflectivity, range to target, atmospheric conditions, and laser detector. A typical value seen on today's laser scanners is $2-5 \mathrm{~cm}(1 \sigma)$ [9] [10] [11]. Thus, the nominal SNR appears to range from $9.5 \mathrm{~dB}$ to $17.5 \mathrm{~dB}$, assuming a pulse rise time of $1 \mathrm{~ns}$.

Scan angle errors do not appear to be addressed in available airborne LiDAR manuals or literature. However, there are specifications on angle measurement resolution, thus it can be assumed a small amount of quantization error will be present. The Riegl LMQ-280 has an angular resolution of $0.0025^{\circ}$ [10]. If it is assumed the encoder measurement is accurate to its resolution then zero mean uniformly distributed quantization errors can be assumed with a standard deviation $0.0007^{\circ}(1 \sigma)$. It will be seen in the IMU Sensor error section that the standard deviation of this angle error is more than a factor of ten less than the standard deviation of the IMU angle error.

\section{Kinematic GPS Sensor Error}

The quality of the kinematic GPS position measurement is dependent on may factors such as satellite geometry measured by the Dilution Of Precision (DOP), multipath, distance between the aircraft and the base station, dynamics of the aircraft, and the post-processing software's ability of to detect and correct GPS carrier phase tracking cycle slips. Typical accuracies given a lowdynamic flight profile are nominally between 5 and $15 \mathrm{~cm} 1 \sigma[12]$.

\section{IMU Sensor Errors}

Current Position and Orientation Systems (POS) typically use medium to high grade IMUs and blend the IMU measurements with kinematic GPS to obtain aircraft attitude. One commonly used POS system is the Applanix POS AV 410 system. Its system specifications are presented to provide a baseline for a POS system. As stated in the product literature, the POS AV 410 is capable of a roll \& pitch error of $0.008^{\circ} \mathrm{RMS}(1 \sigma)$ and a heading error of 0.015 RMS $(1 \sigma)$ after postprocessed GPS/IMU integration [13].

\section{Vertical System Errors}

Within the context of this paper only the vertical components of the LiDAR illumination point are explored, since the primary purpose of this paper is not to describe the error budget of a LiDAR.

Errors in the vertical measurement will be calculated from three components. In these error calculations the following assumptions are made; a max roll of $5^{\circ}$, a max pitch of $5^{\circ}$, and a max scanner angle of $13.9^{\circ}$. Also, changes in the terrain height due to horizontal measurement errors are excluded from these three components. The first vertical error component is the error in the laser scanner range measurement which will be given as $3 \mathrm{~cm}$ $(1 \sigma)$. The second vertical error component is a function of the error in the pitch and roll and the current pitch, roll and scan angle and is given by:

$$
\Delta r=h\left[1-\frac{\cos (\theta+\Delta \theta)}{\cos (\theta)}\right]
$$

where $r$ is the range from the aircraft to the terrain, $\theta$ is the laser angle deviation from nadir (combination of roll, pitch and scan angle), $\Delta \theta$ is the angle measurement error, and $\Delta r$ is the range error due to the angle measurement error [12]. If $\Delta \theta$ is small, this equation can be approximated by:

$$
\Delta r \cong r \Delta \theta \tan (\theta)
$$

where $\Delta \theta$ is in radians [12]. Assuming a scan angle of 13.9 degrees, a max pitch and roll of 5 degrees, and a $\Delta \theta$ of $0.008^{\circ}, 0.14 \mathrm{mrad},(1 \sigma)$, the error component added to the vertical is approximately 7 $\mathrm{cm}(1 \sigma)$. The third vertical error component is from the GPS vertical position error. This will be assumed to be $15 \mathrm{~cm}(1 \sigma)$.

Assuming normal distributions for these errors, they can be combined by root sum squaring the value to get a total vertical error of $17 \mathrm{~cm} 1 \sigma$. As was mentioned before, these errors exclude the effect of horizontal errors leading to a vertical error. These horizontal errors could increase the vertical error component greatly in urban areas. 


\section{LiDAR Error Budget (Real-Time)}

The major improvements in the LiDAR accuracy are obtained by post-processing the GPS and the IMU data. However, in a real-time integrity monitor post-processing is by nature not possible. Therefore, the real-time performance of the GPS/IMU will drive the error standard deviations. The Applanix POS AV 410, real-time DGPS position specification is $0.5-2 \mathrm{~m}$ RMS $(1 \sigma)$ and real-time attitude specification is $0.015^{\circ} \mathrm{RMS}(1 \sigma)$ in the pitch \& roll, and $0.050^{\circ} \mathrm{RMS}(1 \sigma)$ in heading. These numbers will be used in the determination of the Real-Time LiDAR error budget since these systems are currently available. It should be noted new GPS/INS integration schemes have shown the ability to provide similar pitch and roll accuracies of $0.015^{\circ} \mathrm{RMS}(1 \sigma)$ and $0.019^{\circ} \mathrm{RMS}(1 \sigma)$ using a low cost $(<\$ 5 \mathrm{k})$ IMU [14].

Assuming a vertical position error of $2 \mathrm{~m}(1 \sigma)$, a pitch and roll error of $0.015^{\circ}(1 \sigma)$. (which maps to $12.3 \mathrm{~cm}(1 \sigma))$, and a laser range error of $3 \mathrm{~cm}(1 \sigma)$, the total vertical error becomes dominated by the GPS accuracy and equals $2 \mathrm{~m}(1 \sigma)$.

\section{LiDAR Point Cloud Density}

The density of the LiDAR measurements and the size of the area mapped in one pass are dependent on the laser scanner scan rate, scan range, scan pattern, and Pulse Repetition Frequency (PRF); as well as the height Above Ground Level (AGL) and the ground speed of the aircraft.

A $10 \times 18 \mathrm{~km}^{2}$ area of LiDAR data was collected by the National Geodetic Survey (NGS) over Reno, Nevada. This area was centered over the KRNO airport. The LiDAR used was an Optech ALTM 2050 configured with a half scan angle of $13.9^{\circ}$, a scan frequency of $41.3 \mathrm{~Hz}$, and a laser PRF of $50 \mathrm{kHz}$. The data collection was flown at an altitude of $4000 \mathrm{ft}$ AGL with an average ground speed of $83 \mathrm{~m} / \mathrm{s}$ (160 knots). The point cloud density for the database generated can be estimated to be a bit more than $1 \mathrm{~m}$ on the along track and $1 \mathrm{~m}$ on cross track. This estimation was verified by examining the data in the point cloud. This point cloud was then converted to an evenly spaced $2 \mathrm{~m}$ grid. A Matlab surface plot of this data is shown in Figure 2.

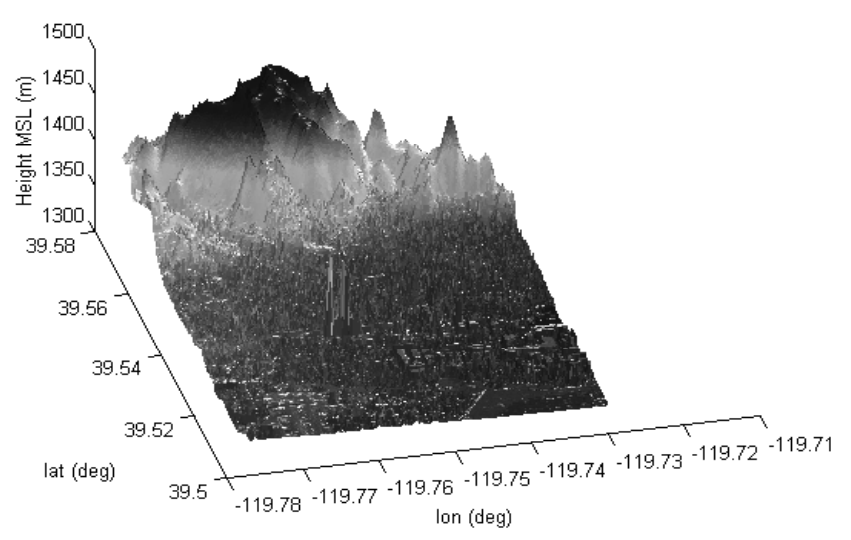

Figure 2, LiDAR Generated DEM, Reno, NV

\section{LiDAR Integrity Monitoring Results}

\section{Disparity characteristics when using LiDAR}

To assess the performance of the LiDAR based integrity monitor, a terrain database was generated as specified in the LiDAR Point Cloud Density section. The post-processed LiDAR point cloud data from one flight over the Reno area was then used to create a synthesized terrain profile. To ensure independence every $100^{\text {th }}$ illumination point was taken. This synthesized terrain profile was then compared to the DEM generated from the LiDAR data. The disparities can be seen in Figure 3.

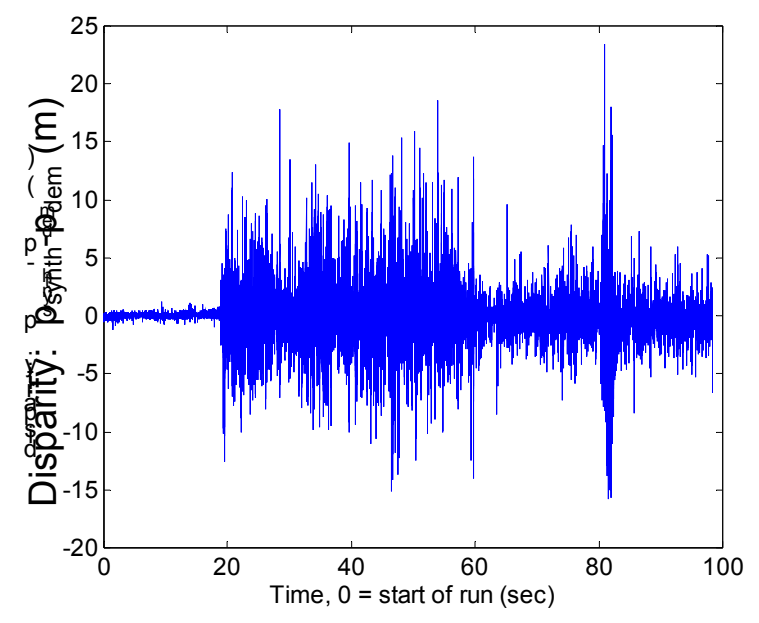

Figure 3. Height Disparities Between LiDAR Sensor and LiDAR DEM. 
It is of interest that during the first 20 seconds of the run, the disparities RMS value of $0.14 \mathrm{~m}$ is near the theoretical RMS value. However, the disparities RMS value increases to $1.26 \mathrm{~m}$ during the last 80 seconds of the run.

Figure 4 provides some insight into this phenomenon. It can be seen that the terrain over which the aircraft is flying becomes significantly rougher at the point where the RMS values of the disparities increase. This increase in roughness is due to the presence of man-made objects such as buildings.

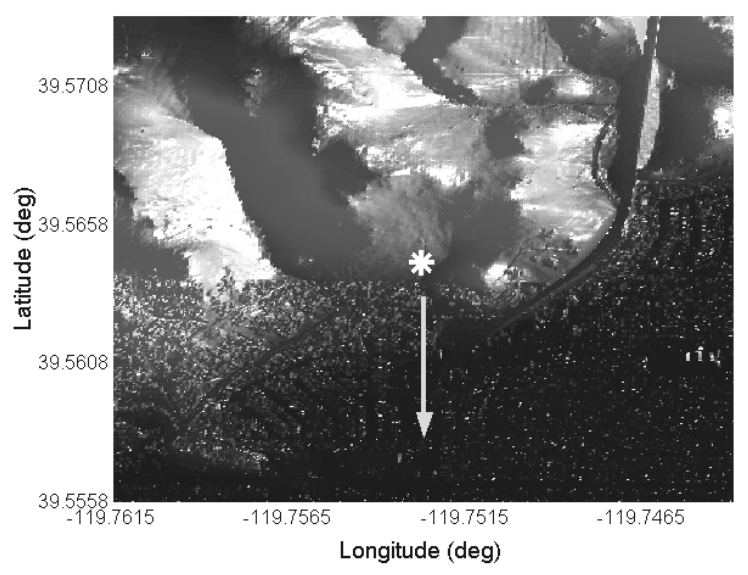

Figure 4. Aircraft Position at Point where Disparities Increase (Aircraft Represented by Asterisk)

As was stated before, the LiDAR point cloud used to generate the synthesized profile is the same LiDAR profile used to generate the $2 \mathrm{~m}$ post spaced DEM, thus the errors present in the disparities can most likely be attributed to interpolation and quantization errors introduced during the DEM generation.

\section{LiDAR Integrity Monitor}

As was shown in the last section, LiDAR operation over smooth terrain leads to a disparity RMS value that closely matches the theoretical vertical error value. LiDAR integrity monitor operation over this smooth terrain will be examined in this section. Three cases will be discussed; the first case uses data collected during the mapping mission; the second case uses the same data with a 1 meter vertical (up) bias added to the aircraft position; and the third case again uses the same data with a 2 meter horizontal (east) bias added to the aircraft position. In these plots, the standard deviation of the disparities is assumed to be $20 \mathrm{~cm}$ $1 \sigma$. The parameters used to generate the $\mathrm{T}$ statistic are as follows: $N=50, P_{F F D}=10^{-4}$, and the time to alert would be $100 \mathrm{~ms}$ given a laser PRF of 50,000 pulses/sec and a 100:1 laser down sampling. Note that this Time-to-Alert is significantly smaller than the Time-to-Alert in the radar altimeter based integrity monitor [2][4].

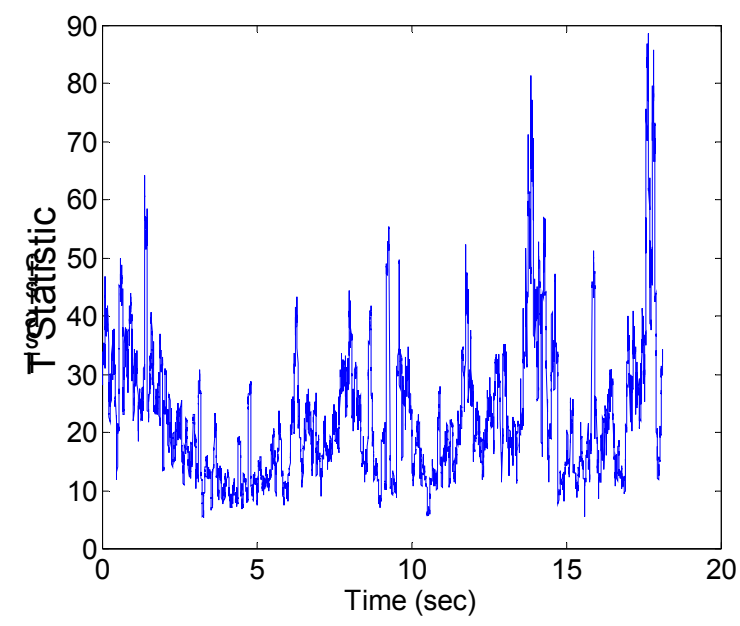

Figure 5, Integrity Monitor Test Static, First 20 Seconds of Run, $T_{D}=96$

Figure 5 shows that over the 20 seconds the integrity monitor is executing, the test statistic threshold, $T_{D}$, is not exceeded. It should be noted that for the next 20 seconds of suburban over-flight data (not shown), $T_{D}$ is greatly exceeded. Figure 6 shows the sensitivity of the integrity monitor to a 1 meter bias in the vertical (up) direction. The $T_{D}$ of 96 is exceeded in the entire dataset. Figure 6 furthermore illustrates the improved sensitivity of the LiDAR-based terrain database integrity monitor to vertical errors as compared to the radar altimeterbased integrity monitor. 


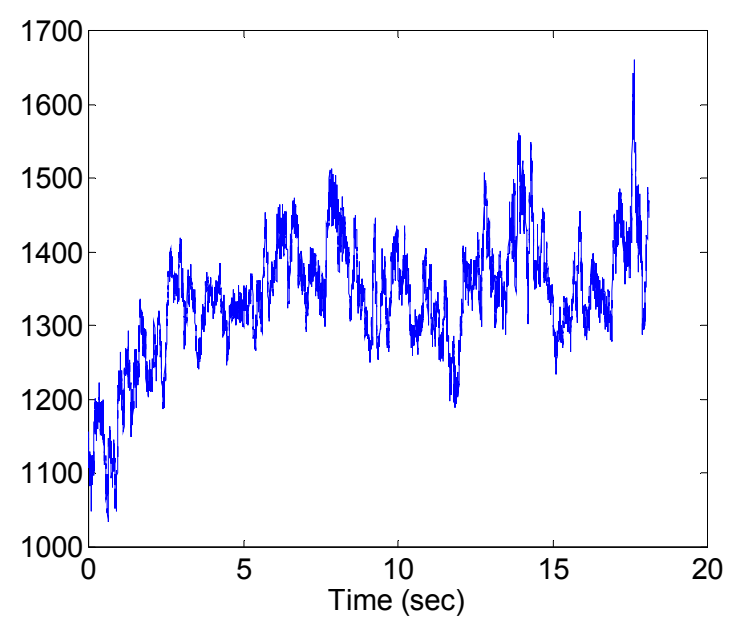

Figure 6, Integrity Monitor Test Statistic Given a $1 \mathrm{~m}$ Up Position Bias Error, $T_{D}=96$

Figure 7 illustrates the effect a 2 meter horizontal bias has on the test statistic. It is clearly not as detectable as a vertical bias, yet it does exceed $T_{D}$ a significant portion of the time. Again, the performance is an improvement over the radar altimeter based system.

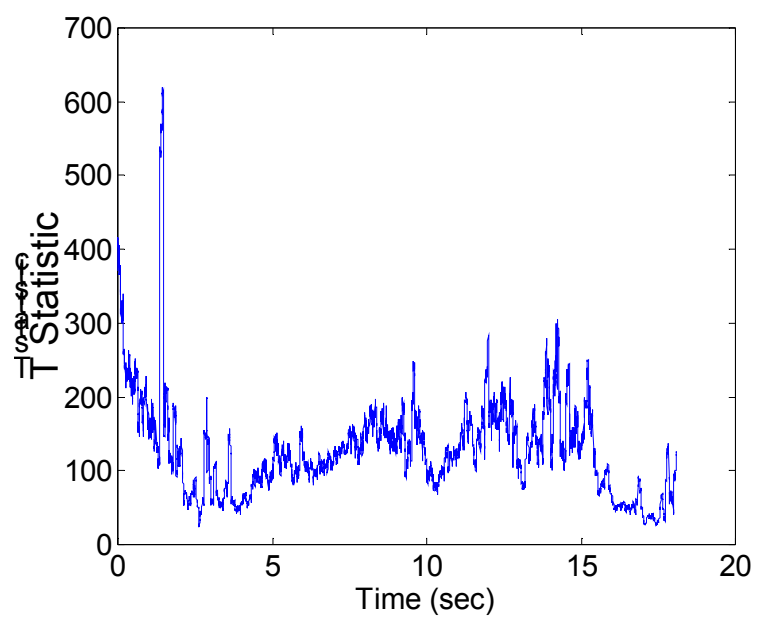

\section{Figure 7, Integrity Monitor Test Statistic Given a 2 m East Position Bias Error, $T_{D}=96$}

It was shown in a previous section that when operating over rougher terrain (suburban area), the RMS value of the disparities increases significantly. When applying the integrity monitor to the flight profile over the rough section of the DEM it is necessary to increase the assumed RMS values of the disparities to $4 \mathrm{~m}$ to maintain zero integrity alerts. Again, this is most likely due to interpolation and quantization errors.

\section{Summary and Conclusions}

The use of LiDAR in a real-time terrain database integrity monitor for a Synthetic Vision System was explored in this paper. Over smooth terrain the LiDAR integrity monitor performed as expected. However it was seen that when operating over rougher terrain, such as over a suburban area, the performance did not match the simple sensor error model given in this paper. One issue is the ability of LiDAR to provide centimeter level point in space measurements. This extremely high accuracy does not appear to be met by a terrain database generated directly from high resolution LiDAR data when performing single pass mapping over man made objects.

This paper only provides an initial analysis of a LiDAR-based terrain database integrity monitor. The results presented were for a time-to-alarm of 0.1 second. The level of integrity provided would increase given a longer time to alarm (the use of more samples in the $T$ value calculation). New statistical distributions and test statistics that exploit the high sampling rate of the LiDAR have yet to be explored. The analysis in this paper indicates improved integrity monitor sensitivity to both vertical and horizontal biases when compared to the radar altimeter-based terrain database integrity monitor.

An area not addressed in this paper is the performance of LiDAR in reduced visibility conditions. Lasers are greatly attenuated under reduced visibility conditions which can occur from fog, clouds, rain, and snow. Future laser technological developments such as adjustable gain and multi-spectral lasers may allow for techniques which reduce the impact of these attenuating factors.

\section{Acknowledgements}

This paper would not have been possible without the help and support of many people. We would like to thank the USGS for making their DEMs available for download. We would also like to thank the pilots and operators at NGS for the flying and processing of the LiDAR terrain 
database over Reno, NV. The work presented in this paper was supported and funded through NASA under Cooperative Agreement NCC-1-351.

\section{References}

[1] Williams, D., et al., January 2001, "Concept of Operations for Commercial and Business Aircraft Synthetic Vision Systems - Version 1.0," NASA Langley Research Center.

[2] Gray, R. A., June 1999, "In-flight Detection of Errors for Enhanced Aircraft Flight Safety and Vertical Accuracy Improvement Using Digital Terrain Elevation Data with an Inertial Navigation System, Global Positioning System and Radar Altimeter," Ph.D. Dissertation, Ohio University, Athens, Ohio.

[3] Young, S., M. Uijt de Haag, J. Sayre, April 2125, 2003, "Using X-band Weather Radar Measurements to Monitor the Integrity of Digital Elevation Models for Synthetic Vision Systems", 17th Annual International Symposium on Aerospace/Defense Sensing, Simulation, and Controls - AeroSense, International Society for Optical Engineering (SPIE), Orlando, Florida.

[4] Campbell, J., M. Uijt de Haag, R. Gray, June 2001, "Availability Aspects of a Terrain Database Integrity Monitor," ION $57^{\text {th }}$ Annual Meeting, Albuquerque, NM.

[5] Uijt de Haag, M., et al., April 2001 "Flight Test Results of a Synthetic Vision Elevation Database Integrity Monitor", Proceedings of the Society of Photo-Optical Instrumentation Engineers (SPIE), Orlando, FL.

[6] Wehr, A., U. Lohr, 1999, “Airborne laser scanning - an introduction and overview," ISPRS Journal of Photogrammetry \& Remote Sensing 54, pp 68-82.
[7] Huising, E.J., L. M. Gomes Pereira, 1998, "Errors and accuracy estimates of laser data acquired by various laser scanning systems for topographic applications," ISPRS Journal of Photogrammetry \& Remote Sensing 53, pp245-261.

[8] American National Standards Institute, 2000, "American National Standard for Safe Use of Lasers", ANSI Z-136.1, American National Standards Institute.

[9] Optech Inc., 2003, “ALTM specifications," Optech Inc., August 13, www.optech.on.ca/altmspecs.htm.

[10] Riegl, 2002, "Laser Mirror Scanner LMS Q280 Technical Documentation and User's Instructions, Preliminary," Riegl Laser Measurement Systems, Austria.

[11] Reigl, 2001, "Laser Mirror Scanner LMSQ140-60-HR, Technical Documentation and User's Instructions," Edition 00-06, Rev 99-06-30, Rieg1 Laser Measurement Systems, Austria.

[12] Baltsavias, E. P., 1999, “Airborne laser scanning: basic relations and formulas," ISPRS Journal of Photogrammetry \& Remote Sensing 54, pp 199-214.

[13] Applanix, 2003, "POS AV 410 Specifications," Applanix, August 14, www.applanix.com/pdf/AV410V4_rev0901_spec\%20sheet.pdf.

[14] van Graas, F., J. L. Farrel, 2001, “GPS/INS A Very Different Way", ION $57^{\text {th }}$ Annual Meeting/GIGTF $20^{\text {th }}$ Biennial Guidance Test Symposium, 11-13 June, Albuquerque, NM. 